\title{
Qualidade de vida de sujeitos com doença de Parkinson e seus cuidadores
}

\section{Quality of life of subjects with Parkinson's disease and caregivers}

\author{
Nadiesca Taisa Filippin ${ }^{[a]}$, Juliana Saibt Martins ${ }^{[b]}$, Lucas Bolzan Dela Libera ${ }^{[c]}$, \\ Bianca Fraga Halberstadt ${ }^{[\mathrm{d}]}$, Alexandre Rodrigues Severo ${ }^{[\mathrm{d}]}$
}

[a] Fisioterapeuta, doutora em Fisioterapia, docente do curso de Fisioterapia do Centro Universitário Franciscano, Santa Maria, RS - Brasil, e-mail: nadifilippin@yahoo.com.br

[b] Fisioterapeuta, doutora em Ciências Biológicas: Bioquímica, docente do curso de Fisioterapia do Centro Universitário Franciscano, Santa Maria, RS - Brasil, e-mail: jsaibt@yahoo.com.br

[c] Fisioterapeuta, aluno do Programa de Residência Multiprofissional em Atenção Básica/Saúde da Família da Universidade do Extremo Sul Catarinense (Unesc), Criciúma, SC - Brasil, e-mail: lucas-bolzan@hotmail.com

[d] Acadêmicos do curso de Fisioterapia do Centro Universitário Franciscano, Santa Maria, RS - Brasil, e-mails: biancahalberstadt@hotmail.com; xandesevero@hotmail.com

\section{Resumo}

Introdução: A doença de Parkinson (DP) interfere na autonomia e independência do sujeito, afetando sua qualidade de vida (QV). Cuidadores podem ter sua QV comprometida, em função das dificuldades com o cuidado. Objetivo: Avaliar e comparar a QV de sujeitos com DP e seus cuidadores e correlacionar a qualidade de vida com as características dos sujeitos. Material e métodos: Foram avaliados 10 sujeitos com DP, com idade média de 65,4 anos, e 8 cuidadores, com idade média de 60,6 anos, de ambos os gêneros. Os instrumentos utilizados foram ficha de avaliação, Questionário PDQ-39, Questionário SF-36, UPDRS e Escala de Hoehn e Yahr modificada. Os dados foram analisados por meio de estatística descritiva e coeficiente de correlação de Pearson e Spearman, utilizando nível de significância de $\alpha$ igual a 5\%. Resultados: A percepção geral sobre a QV foi moderadamente boa tanto para os sujeitos com DP quanto para cuidadores. No entanto, aspectos motores e emocionais influenciam negativamente a QV dos sujeitos com DP. Do contrário, aspectos sociais, mentais e demográficos parecem não exercer influência significativa. Para os cuidadores, fatores emocionais, sociais e físicos, além de características pessoais, estão relacionados com pior percepção sobre sua QV. As características dos sujeitos com DP e aquelas relacionadas ao cuidado não apresentaram correlação significativa com a QV dos cuidadores. Considerações finais: Conhecer os principais fatores que 
interferem na QV ajuda a direcionar estratégias de prevenção, orientação, suporte e tratamento de pessoas com DP e seus cuidadores. A atenção deve ser integral, voltada para um conjunto de aspectos.

Palavras-chave: Qualidade de vida. Doença de Parkinson. Cuidadores.

\section{Abstract}

Background: Parkinson's disease (PD) interferes in the autonomy and independence of the subjects and affects their quality of life (QoL). Due to difficulty with the care, the QoL of caregivers can also be impaired. Purpose: To assess and to compare the QoL of subjects with Parkinson's disease and their caregivers and to correlate quality of life and subjects' characteristics. Material and methods: Ten subjects with PD, mean age of 65.4 years, and eight caregivers, mean age of 60.6 years, both gender, took part in this study. A questionnaire comprising characteristics of the subjects, PDQ-39 questionnaire, SF-36 questionnaire, UPDRS and modified Hoehn and Yahr scale were used to evaluation. Data were analyzed through descriptive statistics, Pearson and Spearman correlation coefficient, using 5\% level of significance. Results: General perception about QoL of subjects with PD and their caregivers was moderately good. However, motor and emotional aspects have a negative impact on QoL of subjects with PD. On the other hand, social, mental and demographic aspects seem not to influence significantly. Emotional, social and physical factors and, personal characteristics are related to worse perception of caregivers about your QoL. Characteristics of the subjects with PD and those related to care not presented significant correlation with QoL of caregivers. Conclusions: To know the main factors that interfere in the QoL helps to address prevention strategies, orientation, support and treatment of subjects with $P D$ and their caregivers. Attention to health should be integral considering a group of aspects.

Keywords: Quality of life. Parkinson's Disease. Caregivers.

\section{Introdução}

A doença de Parkinson (DP) afeta principalmente pessoas a partir da quinta década de vida e pode ser caracterizada como uma doença crônico-degenerativa, que interfere nos movimentos voluntários e automáticos, em virtude de uma disfunção dos gânglios da base. A causa dessa disfunção é a morte das células produtoras de dopamina na parte compacta da substância negra e das células produtoras de acetilcolina no núcleo pedúnculo-pontino $(1,2)$. Os sinais e sintomas mais comuns da DP são rigidez, hipocinesia, bradicinesia, acinesia, tremor de repouso, instabilidade postural e comprometimento visual-perceptivo, além de deficit emocionais e cognitivos (2).

Os tratamentos farmacológicos e cirúrgicos atuais podem atenuar os sintomas e prolongar a vida desses sujeitos, no entanto, aumentam o tempo em que necessitam de cuidados (3). As desordens motoras e não motoras provocadas pela DP levam a quedas, isolamento social, perda de hobbies e atividades de lazer, aumento da dependência para as atividades de vida diária (AVDs), perda de autonomia e consequente redução da qualidade de vida $(4,5,6)$.

Nesse contexto, o papel do cuidador em dirigir atenção, dedicar-se aos cuidados básicos e se responsabilizar por um sujeito com DP torna-se extremamente importante. Contudo, segundo Dick (7), o cuidador apresenta dificuldades em lidar com a variedade de manifestações provocadas pela doença e se vê diante de um futuro incerto. Além disso, conforme as condições do sujeito com DP, o cuidador enfrenta o desafio de ajustar sua vida de acordo com os cuidados que devem ser prestados. Lidar com as dificuldades de execução das AVDs, incapacidades físicas, desordens cognitivas, depressão ou qualquer outra manifestação da doença pode sobrecarregar o cuidador e provocar angústia. Aspectos como rotina, atividades sociais, saúde física e mental podem ser afetados e interferir diretamente na qualidade de vida dos cuidadores $(8,9,10)$. No entanto, a percepção de sujeitos com DP e cuidadores sobre sua qualidade de vida é subjetiva, multidimensional e depende do controle dos sintomas e da progressão da doença (5, 11,12), além de outros aspectos. 
Alguns estudos vêm sendo conduzidos com o objetivo de avaliar a qualidade de vida de sujeitos com DP $(12,13,14)$, seus cuidadores $(8,9,10,15$, $16,17,18,19)$ e ambos os grupos simultaneamente (20). Esses estudos têm identificado que fatores sociais, psicológicos, sociodemográficos e clínicos podem interferir na qualidade de vida desses sujeitos. Especificamente, quanto à qualidade de vida do cuidador, tem-se observado que as características clínicas da doença, as próprias características do cuidador e os aspectos relacionados ao cuidado influenciam nessa percepção.

Assim, compreender em que extensão esses fatores interferem na qualidade de vida desses sujeitos se torna relevante para que se possam propor estratégias de intervenção satisfatórias capazes de diminuir o impacto da doença sobre esses aspectos. Segundo Martínez-Martín (5), além do cuidado com a doença e com o aumento da sobrevida, os profissionais da saúde devem objetivar a garantia da qualidade de vida dos sujeitos com DP. Além disso, é indispensável voltar atenção especial para os cuidadores, pois eles necessitam de assistência, suporte, orientação e cuidado, que poderiam resultar em melhora da qualidade de vida e, segundo McRae e colaboradores (12), influenciar positivamente nos cuidados com o sujeito com DP.

Portanto, os objetivos do estudo foram avaliar e comparar a qualidade de vida de sujeitos com DP e seus cuidadores e correlacionar a qualidade de vida com as características dos sujeitos com DP e cuidadores.

\section{Material e métodos}

0 estudo caracteriza-se como transversal. Foram avaliados dez sujeitos com DP (GP) e oito cuidadores (GC), de ambos os gêneros, com função cognitiva preservada, verificada pelo Mini Exame do Estado Mental (MEEM), pontuado de acordo com o nível de escolaridade (21). A amostra se deu por conveniência e os sujeitos foram recrutados nos serviços de fisioterapia oferecidos pelo Centro Universitário Franciscano e por meio de divulgação em mídia impressa e eletrônica. Foram excluídos da amostra os sujeitos com DP que apresentavam outras doenças neurológicas associadas. Este estudo foi aprovado pelo Comitê de Ética em Pesquisa com Seres Humanos do Centro Universitário Franciscano, Santa
Maria (RS) (Parecer n. 055.2011.2). Todos os sujeitos foram informados sobre os procedimentos do estudo e assinaram um termo de consentimento livre e esclarecido (TCLE). 0 estudo está de acordo com a Resolução n. 466/12 do CNS.

Os instrumentos utilizados para a coleta de dados foram ficha de avaliação, contendo questões sobre dados pessoais e características dos sujeitos com DP e cuidadores; Questionário de Qualidade de Vida na Doença de Parkinson (PDQ-39); Questionário de Avaliação de Qualidade de Vida SF-36; Escala Unificada de Avaliação da Doença de Parkinson (UPDRS) - seções II e III e Escala de Hoehn e Yahr modificada (H-Y modificada). As questões contidas na ficha de avaliação foram geradas e estruturadas a partir da literatura $(9,10,15,16)$.

Quanto aos procedimentos, primeiramente, os sujeitos responderam ao teste MEEM, para avaliação da função cognitiva. Em seguida, foi feita uma entrevista para a coleta dos dados pessoais e das características de cada grupo de sujeitos. Posteriormente, foram aplicados o PDQ-39, SF-36, UPDRS e H-Y modificada para os sujeitos com DP e o SF-36 para os cuidadores.

As características dos sujeitos com DP coletadas foram: idade, gênero, estado civil, escolaridade, idade de início da doença, duração da doença, estágio da doença, quantidade de medicação e renda. As características dos cuidadores englobaram idade, gênero, estado civil, escolaridade, renda, tempo como cuidador, horas gastas com cuidados pessoais, lazer e atividades sociais e horas gastas no cuidado com o paciente. O PDQ-39 é um instrumento específico de avaliação da qualidade de vida de sujeitos com DP e consiste em 39 questões (22). Uma baixa pontuação indica melhor qualidade de vida, segundo a percepção do paciente. O SF-36 é um instrumento genérico de avaliação da qualidade de vida, composto por 36 itens. É pontuado de 0 a 100 , sendo que, quanto mais baixa a pontuação, pior é o estado de saúde (23). A UPDRS, utilizada para identificar o nível de incapacidade dos sujeitos com DP, possui 42 itens, divididos em quatro seções principais, das quais foram avaliadas as seções II e III (atividades de vida diária e atividade motora). A pontuação máxima é indicativa de maior comprometimento pela doença (24). 0 estágio da doença foi avaliado pela Escala H-Y modificada, pontuada de 0 a 5 , sendo que, quanto maior a pontuação, mais grave o comprometimento.

Após a coleta dos dados, os sujeitos interessados foram encaminhados para atendimento 
fisioterapêutico individual ou em grupo no Laboratório de Ensino Prático em Fisioterapia do Centro Universitário Franciscano.

Para a análise dos dados, foi realizada estatística descritiva (média e desvio-padrão). Os dados foram testados quanto à normalidade (teste de ShapiroWilk) e homogeneidade das variâncias (teste de Levene). A comparação da qualidade de vida entre os grupos se deu por meio do teste de MannWithney. O coeficiente de correlação de Spearman foi utilizado para verificar a relação entre a qualidade de vida e as características dos sujeitos do GP e entre a qualidade de vida dos cuidadores e as características dos sujeitos do GP e GC. 0 nível de significância utilizado foi de alfa igual a 5\%. 0 software GB-Stat (v. 5.1, Dynamic Microsystems Inc.) foi usado para a análise estatística.

\section{Resultados}

Na Tabela 1 são apresentadas as características dos sujeitos do Grupo Parkinson (GP) e Grupo Cuidadores (GC).

Nenhum sujeito do GC participava de grupos de apoio até o momento da coleta.

A Figura 1 mostra a qualidade de vida de sujeitos do GP e GC, avaliada pelo questionário SF-36. A comparação entre os escores de ambos os grupos mostrou que houve diferenças estatisticamente significativas no domínio capacidade funcional $(\mathrm{p}=0,01)$, dor $(\mathrm{p}=$ $0,03)$ e estado geral de saúde $(p=0,01)$. Nos demais domínios, não houve diferenças significativas entre os grupos ( $p \geq 0,05)$.

A Figura 2 apresenta o escore total e os subitens do PDQ-39 para o GP.

Tabela 1 - Características dos sujeitos

\begin{tabular}{lcc}
\hline Características & GP $(\mathbf{n}=10)$ & GC $(\mathbf{n}=\mathbf{8})$ \\
\hline Idade (anos) & $65,40( \pm 8,53)$ & $60,63( \pm 17,69)$ \\
Gênero (M/F) & $3 / 7$ & $2 / 6$ \\
Escolaridade (anos) & $6,90( \pm 4,95)$ & $10,00( \pm 5,13)$ \\
Estado civil (S/C/D/N) & $0 / 5 / 1 / 4$ & $1 / 3 / 1 / 3$ \\
Renda (em salários) & $4,75( \pm 3,54)$ & $2,75( \pm 1,83)$ \\
Número de pessoas na casa & $2,70( \pm 1,34)$ & - \\
Idade de início dos sintomas (anos) & $57,10( \pm 10,34)$ & - \\
Duração da doença (anos) & $9,30( \pm 6,20)$ & - \\
Estágio da doença & $2,60( \pm 0,74)$ & - \\
Quantidade de medicamentos & $4,80( \pm 2,53)$ & - \\
Tratamento fisioterapêutico (Sim/Não) & $7 / 3$ & - \\
UPDRS AVDs & $13,20( \pm 6,18)$ & - \\
UPDRS motora & $15,60( \pm 7,47)$ & $47,75( \pm 50,64)$ \\
Cuidador (Sim/Não) & $8 / 2$ & $8,00( \pm 5,93)$ \\
Tempo como cuidador (meses) & - & $10,25( \pm 7,50)$ \\
Horas gastas com cuidados pessoais (diárias) & - & - \\
Horas gastas nos cuidados com paciente (diárias) & - & - \\
\hline
\end{tabular}

Legenda: $\mathrm{M}=$ masculino; $\mathrm{F}=$ feminino; $\mathrm{S}=$ solteiro; $\mathrm{C}=$ casado; $\mathrm{D}=$ divorciado; $\mathrm{V}=$ viúvo.

Fonte: Dados da pesquisa.

Nota: Dados expressos em média ( \pm desvio-padrão). 


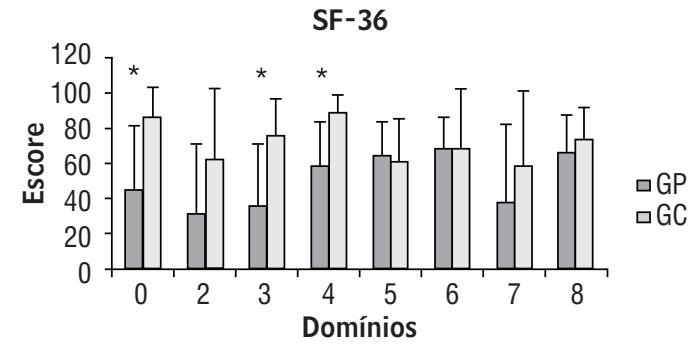

Figura 1 - Qualidade de vida de sujeitos GP $(n=8)$ e GC $(\mathrm{n}=8)-$ SF-36

Legenda: 1 = Capacidade Funcional; 2 = Limitação por Aspectos

Físicos; 3 = Dor; 4 = Estado Geral de Saúde; $5=$ Vitalidade; $6=$ Aspectos Sociais; 7 = Limitação por Aspectos Emocionais; 8 = Saúde Mental

*Significativo em $p \leq 0,05$ (teste de Mann-Whitney).

Fonte: Dados da pesquisa.

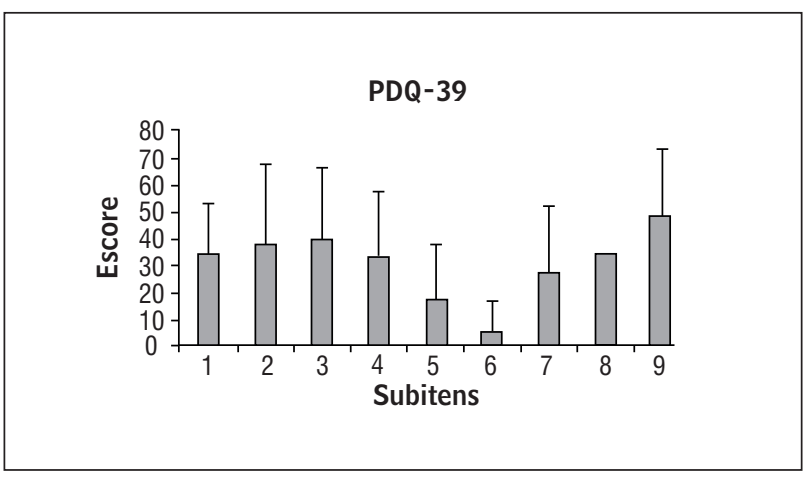

Figura 2 - Qualidade de vida de sujeitos GP - PDQ-39

Legenda: 1 = Escore total; 2 = Mobilidade; $3=$ AVDs; $4=$ Emocional; $5=$ Estigma; $6=$ Apoio Social; $7=$ Cognição; $8=$ Comunicação; 9 = Desconforto Corporal.

Fonte: Dados da pesquisa.

As seguintes características dos sujeitos do GP foram correlacionadas com sua qualidade de vida (escore total da PDQ-39): escolaridade, idade, gênero, estado civil, renda, número de pessoas na casa, idade de início dos sintomas, estágio da doença, duração da doença, quantidade de medicamentos, tratamento fisioterapêutico, cuidador, UPDRS AVDs, UPDRS motora. Foi observado que a qualidade de vida apresentou correlação estatisticamente significativa com a renda familiar dos sujeitos ( $r=0,79 ; p=0,01)$, UPDRS AVDs $(\mathrm{r}=0,64 ; \mathrm{p}=0,00)$ e UPDRS motora $(\mathrm{r}=0,61 ; \mathrm{p}=0,00)$. Houve uma tendência à correlação entre qualidade de vida e estágio da doença $(r=0,60 ; p=0,07)$. As demais características do grupo não mostraram correlação significativa com a qualidade de vida $(p \geq 0,05)$.

A Tabela 2 apresenta a correlação entre a qualidade de vida do GC (avaliada pelo questionário SF-36) e as características dos sujeitos do GC e aquelas relacionadas aos cuidados com o paciente. Houve correlação estatisticamente significativa entre a capacidade funcional e a idade dos sujeitos; entre limitação por aspectos físicos e aspectos sociais com escolaridade; e entre limitação por aspectos emocionais e gênero dos cuidadores. As demais correlações não foram estatisticamente significativas ( $p \geq 0,05)$.

Na Tabela 3 são apresentadas as correlações entre qualidade de vida do GC (avaliada pelo questionário SF-36) e as características dos sujeitos do GP. Foi observada correlação significativa e inversamente proporcional entre quantidade de medicamentos ingerida pelos sujeitos com DP e alguns aspectos da qualidade de vida do cuidador (capacidade funcional, limitação por aspectos físicos, dor, vitalidade e saúde mental).

\section{Discussão}

O presente estudo avaliou e comparou a qualidade de vida de sujeitos com DP e seus cuidadores e correlacionou a qualidade de vida com as características desses dois grupos de sujeitos.

Os sujeitos com DP avaliados se encontram nos estágios leve a moderado da doença, em que há uma manifestação bilateral dos sintomas, progredindo para instabilidade postural; no entanto, o sujeito pode viver independentemente. A maioria dos sujeitos avaliados em ambos os grupos foi do gênero feminino, com idade média superior a 60 anos, corroborando estudos de Lökk (15), Martínez-Martín e colaboradores (17) e Carter e colaboradores (18). Quanto ao estado civil, a maioria dos sujeitos era viúvo ou casado em ambos os grupos. Os cuidadores mostraram maior grau de escolaridade quando comparados aos sujeitos do GP, sendo alguns com Ensino Superior completo. No entanto, a maioria dos sujeitos em ambos os grupos apresentou Ensino Fundamental ou Médio. As características sobre estado civil e escolaridade do presente estudo estão de acordo com o estudo de Martínez-Martín e colaboradores (9).

A quantidade de horas gastas pelo cuidador com os cuidados pessoais, em média, foi menor que as horas gastas no cuidado ao paciente. 0 cuidador precisa fazer 
Filippin NT, Martins JS, Dela Libera LB, Halberstadt BF, Severo AR.

Tabela 2 - Correlação entre qualidade de vida (SF-36) do GC com características dos sujeitos GC

\begin{tabular}{|c|c|c|c|c|c|c|c|c|}
\hline \multicolumn{2}{|c|}{ Domínio/Características } & \multirow{2}{*}{$\begin{array}{r}\text { Idade } \\
-0,75 \\
0,03^{*}\end{array}$} & \multirow{2}{*}{$\begin{array}{c}\text { Gênero } \\
0,13 \\
0,76\end{array}$} & \multirow{2}{*}{$\begin{array}{l}\begin{array}{c}\text { Estado } \\
\text { Civil }\end{array} \\
0,00 \\
1\end{array}$} & \multirow{2}{*}{$\begin{array}{c}\text { Escolaridade } \\
-0,39 \\
0,34\end{array}$} & \multirow{2}{*}{$\begin{array}{c}\begin{array}{c}\text { Tempo } \\
\text { Cuidador }\end{array} \\
0,22 \\
0,60\end{array}$} & \multirow{2}{*}{$\begin{array}{c}\begin{array}{c}\text { Horas cuidados } \\
\text { pessoais }\end{array} \\
-0,11 \\
0,79\end{array}$} & \multirow{2}{*}{$\begin{array}{c}\begin{array}{c}\text { Horas } \\
\text { gastas } \\
\text { paciente }\end{array} \\
-0,20 \\
0,64\end{array}$} \\
\hline Capacidade Funcional & $\begin{array}{l}r \\
p\end{array}$ & & & & & & & \\
\hline $\begin{array}{l}\text { Limitação - aspectos } \\
\text { físicos }\end{array}$ & $\begin{array}{l}r \\
p\end{array}$ & $\begin{array}{r}-0,66 \\
0,08\end{array}$ & $\begin{array}{l}0,26 \\
0,53\end{array}$ & $\begin{array}{l}0,03 \\
0,95\end{array}$ & $\begin{array}{l}-0,72 \\
0,04^{*}\end{array}$ & $\begin{array}{l}0,04 \\
0,92\end{array}$ & $\begin{array}{r}-0,09 \\
0,83\end{array}$ & $\begin{array}{r}-0,12 \\
0,77\end{array}$ \\
\hline Dor & $\begin{array}{l}r \\
p\end{array}$ & $\begin{array}{r}-0,31 \\
0,45\end{array}$ & $\begin{array}{l}0,13 \\
0,76\end{array}$ & $\begin{array}{l}0,20 \\
0,63\end{array}$ & $\begin{array}{l}0,32 \\
0,44\end{array}$ & $\begin{array}{l}0,01 \\
0,99\end{array}$ & $\begin{array}{l}0,09 \\
0,84\end{array}$ & $\begin{array}{r}-0,40 \\
0,33\end{array}$ \\
\hline Estado geral & $\begin{array}{l}r \\
p\end{array}$ & $\begin{array}{l}0,22 \\
0,59\end{array}$ & $\begin{array}{r}-0,26 \\
0,54\end{array}$ & $\begin{array}{l}0,28 \\
0,50\end{array}$ & $\begin{array}{r}-0,45 \\
0,27\end{array}$ & $\begin{array}{r}-0,18 \\
0,66\end{array}$ & $\begin{array}{r}-0,24 \\
0,57\end{array}$ & $\begin{array}{r}-0,02 \\
0,97\end{array}$ \\
\hline Vitalidade & $\begin{array}{l}r \\
p\end{array}$ & $\begin{array}{r}-0,48 \\
0,23\end{array}$ & $\begin{array}{l}0,44 \\
0,27\end{array}$ & $\begin{array}{l}0,45 \\
0,26\end{array}$ & $\begin{array}{r}-0,44 \\
0,27\end{array}$ & $\begin{array}{r}-0,35 \\
0,39\end{array}$ & $\begin{array}{r}-0,10 \\
0,82\end{array}$ & $\begin{array}{r}-0,05 \\
0,91\end{array}$ \\
\hline Aspectos sociais & $\begin{array}{l}r \\
p\end{array}$ & $\begin{array}{r}-0,68 \\
0,07\end{array}$ & $\begin{array}{l}0,52 \\
0,19\end{array}$ & $\begin{array}{l}0,05 \\
0,90\end{array}$ & $\begin{array}{l}-0,77 \\
0,02^{*}\end{array}$ & $\begin{array}{l}0,07 \\
0,86\end{array}$ & $\begin{array}{r}-0,29 \\
0,48\end{array}$ & $\begin{array}{l}0,14 \\
0,75\end{array}$ \\
\hline $\begin{array}{l}\text { Limitação - aspectos } \\
\text { emocionais }\end{array}$ & $\begin{array}{l}r \\
p\end{array}$ & $\begin{array}{r}-0,52 \\
0,18\end{array}$ & $\begin{array}{l}0,78 \\
0,02^{\star}\end{array}$ & $\begin{array}{l}0,13 \\
0,58\end{array}$ & $\begin{array}{r}-0,59 \\
0,13\end{array}$ & $\begin{array}{r}-0,12 \\
0,78\end{array}$ & $\begin{array}{r}-0,34 \\
0,41\end{array}$ & $\begin{array}{l}0,22 \\
0,59\end{array}$ \\
\hline Saúde mental & $\begin{array}{l}r \\
p\end{array}$ & $\begin{array}{r}-0,46 \\
0,26\end{array}$ & $\begin{array}{l}0,50 \\
0,20\end{array}$ & $\begin{array}{l}0,50 \\
0,21\end{array}$ & $\begin{array}{r}-0,38 \\
0,36\end{array}$ & $\begin{array}{r}-0,39 \\
0,35\end{array}$ & $\begin{array}{r}-0,11 \\
0,80\end{array}$ & $\begin{array}{r}-0,02 \\
0,96\end{array}$ \\
\hline
\end{tabular}

Legenda: ${ }^{*}=$ Significativo em $p \leq 0,05$ (coeficiente de correlação de Spearman).

Fonte: Dados da pesquisa.

Tabela 3 - Correlação entre qualidade de vida (SF-36) do GC com características dos sujeitos GP

\begin{tabular}{|c|c|c|c|c|c|c|}
\hline \multicolumn{2}{|c|}{ Domínio/Características } & \multirow{2}{*}{$\begin{array}{l}\text { Idade } \\
\begin{array}{l}0,00 \\
1\end{array}\end{array}$} & \multirow{2}{*}{$\begin{array}{r}\begin{array}{r}\text { Início dos } \\
\text { sintomas }\end{array} \\
-0,25 \\
0,56\end{array}$} & \multirow{2}{*}{$\begin{array}{c}\begin{array}{c}\text { Estágio } \\
\text { doença }\end{array} \\
0,26 \\
0,50\end{array}$} & \multirow{2}{*}{$\begin{array}{c}\begin{array}{c}\text { Duração } \\
\text { doença }\end{array} \\
0,33 \\
0,43\end{array}$} & \multirow{2}{*}{$\begin{array}{c}\begin{array}{c}\text { Quantidade } \\
\text { medicamentos }\end{array} \\
-0,90 \\
0,002^{*}\end{array}$} \\
\hline Capacidade Funcional & $\begin{array}{l}r \\
p\end{array}$ & & & & & \\
\hline $\begin{array}{l}\text { Limitação - aspectos } \\
\text { físicos }\end{array}$ & $\begin{array}{l}r \\
p\end{array}$ & $\begin{array}{l}0,20 \\
0,63\end{array}$ & $\begin{array}{r}-0,04 \\
0,92\end{array}$ & $\begin{array}{r}-0,02 \\
0,96\end{array}$ & $\begin{array}{l}0,38 \\
0,35\end{array}$ & $\begin{array}{l}-0,79 \\
0,02^{\star}\end{array}$ \\
\hline Dor & $\begin{array}{l}r \\
p\end{array}$ & $\begin{array}{r}-0,37 \\
0,36\end{array}$ & $\begin{array}{l}0,56 \\
0,15\end{array}$ & $\begin{array}{l}0,18 \\
0,67\end{array}$ & $\begin{array}{l}0,26 \\
0,54\end{array}$ & $\begin{array}{l}-0,84 \\
0,009 *\end{array}$ \\
\hline Estado geral & $\begin{array}{l}r \\
p\end{array}$ & $\begin{array}{l}0,31 \\
0,46\end{array}$ & $\begin{array}{r}-0,02 \\
0,97\end{array}$ & $\begin{array}{l}0,043 \\
0,92\end{array}$ & $\begin{array}{l}0,62 \\
0,10\end{array}$ & $\begin{array}{r}-0,68 \\
0,06\end{array}$ \\
\hline Vitalidade & $\begin{array}{l}r \\
p\end{array}$ & $\begin{array}{l}0,10 \\
0,82\end{array}$ & $\begin{array}{r}-0,01 \\
0,98\end{array}$ & $\begin{array}{r}-0,21 \\
0,62\end{array}$ & $\begin{array}{l}0,25 \\
0,55\end{array}$ & $\begin{array}{l}-0,79 \\
0,02^{\star}\end{array}$ \\
\hline Aspectos sociais & $\begin{array}{l}r \\
p\end{array}$ & $\begin{array}{l}0,4 \\
0,33\end{array}$ & $\begin{array}{l}0,21 \\
0,62\end{array}$ & $\begin{array}{l}0,13 \\
0,77\end{array}$ & $\begin{array}{l}0,23 \\
0,59\end{array}$ & $\begin{array}{r}-0,54 \\
0,09\end{array}$ \\
\hline $\begin{array}{l}\text { Limitação - aspectos } \\
\text { emocionais }\end{array}$ & $\begin{array}{l}r \\
p\end{array}$ & $\begin{array}{l}0,35 \\
0,39\end{array}$ & $\begin{array}{l}0,29 \\
0,48\end{array}$ & $\begin{array}{l}0,04 \\
0,92\end{array}$ & $\begin{array}{r}-0,05 \\
0,90\end{array}$ & $\begin{array}{r}-0,54 \\
0,16\end{array}$ \\
\hline Saúde mental & $\begin{array}{l}r \\
p\end{array}$ & $\begin{array}{l}0,07 \\
0,86\end{array}$ & $\begin{array}{l}0,00 \\
1\end{array}$ & $\begin{array}{r}-0,21 \\
0,62\end{array}$ & $\begin{array}{l}0,18 \\
0,66\end{array}$ & $\begin{array}{l}-0,77 \\
0,02^{*}\end{array}$ \\
\hline
\end{tabular}

Legenda: * Significativo em $p \leq 0,05$ (coeficiente de correlação de Spearman).

Fonte: Dados da pesquisa. 
contínuos ajustes em sua vida pessoal e profissional, de acordo com o cuidado que deve prestar (7), o que pode produzir danos à sua saúde física e mental. Ele deixa de dedicar seu tempo para lazer, saúde e atividades sociais em prol do cuidado com o outro. Chamou atenção, neste estudo, o fato de que nenhum dos cuidadores participa de grupos de apoio, o que poderia servir de suporte no enfrentamento dos problemas gerados pelo ato de cuidar. Para McRae e colaboradores (10), cuidadores que frequentam grupos de apoio podem lidar melhor com as situações e ajudar os outros a lidarem com circunstâncias semelhantes.

A avaliação do nível de incapacidade dos sujeitos com DP relacionado à atividade motora e AVDs, por meio da UPDRS, mostrou um comprometimento leve. A UPDRS (seções II e III) e a escala de H-Y podem apresentar resultados semelhantes, como no presente estudo, pois avaliam os sinais e sintomas da doença relacionados aos aspectos motores. Comprometimentos motores, embora leves, podem comprometer a qualidade de vida de sujeitos com DP, conforme será discutido adiante.

Em relação à avaliação da qualidade de vida por meio de um questionário genérico (SF-36), foi possível observar que o GP apresentou pior percepção na maioria dos domínios, quando comparado ao GC, sendo que as diferenças estatisticamente significativas foram encontradas para capacidade funcional, dor e estado geral de saúde. Avaliando-se individualmente os escores para o GP, pode-se observar que tanto aspectos físicos quanto emocionais, em maior ou menor proporção, afetam a percepção que os sujeitos têm de sua qualidade de vida. Apesar de aspectos motores clássicos da doença que dificultam as atividades e aumentam a dependência parecerem mais significativos na autoavaliação da qualidade de vida do presente estudo, é reconhecido que manifestações não motoras têm grande impacto na vida de sujeitos com DP (25).

Por outro lado, os sujeitos com DP apresentaram percepção semelhante à dos cuidadores nos aspectos sociais, saúde mental e vitalidade. Isso indica que a doença, por si só, pode não interferir nesses aspectos, considerando o estágio em que se encontram os sujeitos do GP. De acordo com Kuopio e colaboradores (26), a perda da função social está relacionada com fatores como idade, estágios da doença e comprometimento cognitivo, sendo que a progressão da doença resulta em comprometimentos físicos, mentais e emocionais.

Quanto aos cuidadores, a pior percepção sobre sua qualidade de vida apareceu nos aspectos emocionais.
Entretanto, de maneira geral, houve boa percepção da qualidade de vida, com ênfase no estado geral de saúde e capacidade funcional. Talvez os cuidadores se vejam melhores que os pacientes quanto à saúde e consideram que precisam estar aptos fisicamente para lidar com eles. Lökk (15) identificou que as condições gerais de saúde dos cuidadores eram satisfatórias, independente da duração da doença. Mesmo assim, observou-se o impacto provocado pelo exercício do cuidar sobre os aspectos emocionais, sociais, de vitalidade e até mesmo físicos. Martínez-Martín e colaboradores (17) descrevem que o estado afetivo é o fator que mais influencia a percepção da sobrecarga e da saúde dos cuidadores. Lökk (15) aponta que cuidar de um paciente com DP tem um impacto negativo significativo sobre muitos aspectos da saúde física e domínios psicossociais do cuidador, resultando em isolamento, sofrimento e ônus. Glozman (27) e McRae e colaboradores (10) observam que a rotina diária, atividades sociais, saúde física e emocional do cuidador são afetadas. Os desafios presentes e a preocupação com o futuro, as perdas, o cansaço, o esgotamento, o desânimo, a ansiedade e a falta de tempo para atividades sociais e de lazer configuram características importantes para a percepção sobre a qualidade de vida dos cuidadores.

Considerando-se o questionário específico da qualidade de vida de sujeitos com DP (PDQ-39), foi possível observar, de maneira geral, uma percepção moderadamente boa, corroborando o estudo de Lana e colaboradores (13). A melhor percepção foi encontrada no subitem relacionado aos aspectos sociais. Isso reforça a ideia de que a questão social, pelo menos nos estágios iniciais e intermediários da doença, pode não ser o aspecto principal, quando se considera a percepção sobre a qualidade de vida, de acordo com o que já foi discutido. Conforme esperado e já discutido, os aspectos físicos (desconforto corporal, mobilidade e AVDs) apresentaram os maiores escores, indicando pior percepção. Filippin, Lobo da Costa e Mattioli (28) encontraram pior percepção nos aspectos mobilidade e AVDs e melhor percepção no item suporte social. Silva e colaboradores (12) e Lana e colaboradores (13) também encontraram maior comprometimento nos aspectos motores (mobilidade e AVDs). Embora apresentem construções diferenciadas, ambos os questionários (SF-36 e PDQ-39) representaram os itens de maior e menor relevância para a qualidade de vida de sujeitos com DP.

O escore total do PDQ-39 apresentou correlação significativa com a renda do GP, indicando que, quanto 
maior a renda, pior a percepção da qualidade de vida. Melhores condições econômicas, acesso à informação, conhecimento e cultura podem tornar o indivíduo mais exigente quanto a seu bem-estar, o que poderia explicar os resultados encontrados. Houve correlação diretamente proporcional entre qualidade de vida e UPDRS (AVDs e motora), ou seja, quanto maior o comprometimento motor, pior a percepção sobre a qualidade de vida. Deve-se ressaltar que os aspectos motores apresentaram os piores valores na avaliação por subitem da qualidade de vida. Por outro lado, Filippin, Lobo da Costa e Mattioli (28) não encontraram associação entre o escore geral da PDQ-39 e a UPDRS motora. Ellis e colaboradores (14) sugerem que testes de função física apresentam valor maior em predizer a qualidade de vida relacionada à saúde do que testes de comprometimentos motores, como a UPDRS.

Houve uma tendência à correlação diretamente proporcional entre qualidade de vida e estágio da doença, ou seja, quanto maior o estágio da doença, maiores as complicações motoras e, consequentemente, pior a percepção sobre a qualidade de vida. A classificação do estágio da doença se baseia em aspectos motores. No presente estudo, esses aspectos se mostraram importantes para os sujeitos como fatores limitantes a uma vida com qualidade. No entanto, para Behari, Srivastava, Pandey (4) e Schrag, Jahanshahi e Quinn (29), o PDQ-39 e as escalas clínicas avaliam diferentes aspectos da DP e a UPDRS e H-Y podem não ser sensíveis para avaliar o impacto da severidade da doença na vida diária.

Aspectos da qualidade de vida dos cuidadores apresentaram relação com algumas de suas características, tais como idade, gênero e escolaridade. Conforme Habermann, Davis (30) e Glozman (27), características pessoais, além daquelas relacionadas ao paciente e ao ambiente, interferem na percepção sobre a saúde e qualidade de vida dos cuidadores.

Quanto maior a idade, pior a percepção sobre sua capacidade funcional. Isso indica que sujeitos mais velhos, pelas modificações comuns ao envelhecimento e pela sobrecarga física gerada pelo cuidado, sentem com maior intensidade o declínio em suas funções. 0 gênero apresentou correlação com os aspectos emocionais, sendo o masculino aquele que apresentou pior percepção nesse aspecto. Tal resultado pode ser explicado pelo fato de que, histórica e culturalmente, as mulheres estão mais habituadas com o ato de cuidar do outro, sendo para os homens uma tarefa complexa, que pode modificar, dentre outros aspectos, sua estrutura emocional. Ainda, quanto maior a escolaridade do cuidador, pior a percepção que ele apresenta sobre os aspectos físicos e sociais, ou seja, quanto maior o grau de instrução, mais crítico e exigente se torna, conseguindo distinguir claramente os aspectos de sua vida que geram repercussões positivas daqueles que geram repercussões negativas.

Por outro lado, características como: estado civil, que poderia representar companheirismo (para os casados) ou sentimento de solidão (para os viúvos, solteiros ou separados), tempo como cuidador, tempo gasto nos cuidados com o paciente e menor tempo para cuidados pessoais, que poderiam indicar desgaste, sobrecarga e adaptações da vida pessoal e profissional, parecem não interferir de maneira significativa na qualidade de vida dos cuidadores avaliados.

Quando se correlacionou a qualidade de vida dos cuidadores com características dos sujeitos do GP, observou-se que, quanto maior a quantidade de medicamentos ingeridos pelo paciente, pior a percepção sobre a capacidade funcional, limitação por aspectos físicos, dor, vitalidade e saúde mental do cuidador. Assim, a qualidade de vida do cuidador está intimamente relacionada com esse aspecto. Em contrapartida, idade, início dos sintomas, estágio da doença e duração da doença dos pacientes do GP parecem não interferir significativamente na qualidade de vida do cuidador. Para Lökk (10), a lembrança frequente de que o paciente deve tomar a medicação da DP é um fator limitante e estressante. Uma explicação complementar para os resultados do presente estudo é que se supõe que, quanto maior a quantidade de medicamentos, maiores são os comprometimentos provocados pela DP, o que torna mais difícil o cuidado. A influência de aspectos não motores poderia explicar por que o estágio da doença não apresentou correlação com a qualidade de vida dos cuidadores. Para Aarsland e colaboradores (31), sintomas mentais, como depressão, prejuízo cognitivo e ilusões, são preditores consistentes da angústia dos cuidadores. Outra explicação seria que somente em estágios mais avançados da doença essa variável teria influência sobre a percepção dos cuidadores. Ao contrário dos resultados do presente estudo, Schestatsky e colaboradores (20) mostraram que a idade do sujeito com DP é um fator preditor dos aspectos sociais da qualidade de vida dos cuidadores.

0 estudo apresentou algumas limitações. 0 número amostral foi reduzido e a seleção dos participantes se deu por conveniência; desse modo, deve-se considerar que os sujeitos deste estudo podem ser 
diferentes de alguma forma da população geral, restringindo a amplitude e a variabilidade da amostra. Além disso, a avaliação de sujeitos com DP em estágios semelhantes da doença e o desenho transversal do estudo restringem a interpretação dos resultados. Assim, os resultados apresentados não podem ser generalizados.

\section{Considerações finais}

Os principais resultados encontrados foram percepção geral moderadamente boa da qualidade de vida, tanto para os sujeitos com DP quanto para cuidadores. No entanto, alguns aspectos parecem interferir negativamente na qualidade de vida do GP e GC. Aspectos motores e emocionais são os principais influenciadores da qualidade de vida dos sujeitos com DP. Já aspectos sociais, mentais e demográficos parecem não exercer influência significativa. Em relação aos cuidadores, a pior percepção sobre sua qualidade de vida esteve relacionada a fatores emocionais, sociais e físicos, além de características pessoais (idade, gênero e escolaridade). Características dos sujeitos do GP e aquelas relacionadas ao cuidado não apresentaram correlação significativa com a qualidade de vida dos cuidadores. Dessa forma, o trabalho de uma equipe multidisciplinar e o desenvolvimento de estratégias de prevenção, orientação, suporte e tratamento de pessoas com DP e seus cuidadores poderiam contribuir para a melhora na qualidade de vida, nos seus mais diversos níveis. 0 conhecimento e a compreensão dos reais aspectos que interferem na percepção da qualidade de vida relacionada à saúde aperfeiçoam e direcionam o trabalho dos profissionais da saúde. Para os sujeitos com DP, não basta apenas dirigir atenção aos aspectos físicos da doença, mas também às demais dimensões que parecem interferir tanto quanto ou mais que os comprometimentos motores, garantindo que estes tenham uma sobrevida com mais qualidade, podendo manter as relações sociais por mais tempo. Em especial, deve-se considerar a participação dos cuidadores em grupos de apoio para que eles compartilhem experiências e sentimentos, aprendam estratégias de enfrentamento e mantenham a saúde física e mental, o convívio social e outras atividades para além do cuidado com o sujeito com DP.

\section{Agradecimentos}

Agradecemos os participantes desta pesquisa, pela disponibilidade e colaboração e ao Centro Universitário Franciscano e à FAPERGS, pelo apoio financeiro.

\section{Referências}

1. Delong MR, Wichmann MD. Circuits and circuit disorders of the basal ganglia. Arch Neurol. 2007;64(1):20-4.

2. Rivlin-Etzion M, Marmor O, Heimer G, Raz A, Nini A, Bergman H. Basal ganglia oscillations and pathophysiology of movement disorders. Curr Opin Neurobiol. 2006;16(6):629-37.

3. McRae C, Sherry P, Roper K. Stress and family functioning among caregivers of persons with Parkinson's disease. Parkinsonism Relat Disord. 1999;5(1-2):69-75.

4. Behari M, Srivastava AK, Pandey RM. Quality of life in patients with Parkinson's disease. Parkinsonism Relat Disord. 2005;11(4):221-6.

5. Martínez-Martín P. An introduction to the concept of "quality of life in Parkinson's disease". J Neurol. 1998;245(Suppl 1):S2-6.

6. Morris ME. Movement disorders in people with Parkinson disease: a model for physical therapy. Phys Ther. 2000;80(6):578-97.

7. Dick C. Who cares for the caregivers? Parkinsonism Relat Disord. 2009;15(Suppl 3):S118-21.

8. Lökk J. Reduced life-space of non-professional caregivers to Parkinson's disease patients with increased disease duration. Clin Neurol Neurosurg. 2009; 111(7):583-7.

9. Martínez-Martín P, Forjaz MJ, Frades-Payo B, Rusiñol AB, Fernández-García JM, Benito-León J, et al. Caregiver burden in Parkinson's disease. Mov Disord. 2007;22(7):924-31.

10. McRae C, Fazio E, Hartsock G, Kelley L, Urbanski S, Russell D. Predictors of loneliness in caregivers of persons with Parkinson's disease. Parkinsonism Relat Disord. 2009;15(8):554-7. 
11. Dowding CH, Shenton CL, Salek SS. A review of the health-related quality of life and economic impact of Parkinson's disease. Drugs Aging. 2006;23(9):693-721.

12. Silva JAMG, Dibai Filho AV, Faganello FR. Mensuração da qualidade de vida de indivíduos com a doença de Parkinson por meio do questionário PDQ-39. Fisioter Mov. 2011;24(1):141-6.

13. Lana RC, Álvares LMRS, Nasciutti-Prudente C, Goulart FRP, Teixeira-Salmela LF, Cardoso FE. Percepção da qualidade de vida de indivíduos com doença de Parkinson através do PDQ-39. Rev Bras Fisioter. 2007;11(5):397-402.

14. Ellis T, Cavanaugh JT, Earhart GM, Ford MP, Foreman KB, Dibble LE. Which measures of physical function and motor impairment best predict quality of life in Parkinson's disease? Parkinsonism Relat Disord. 2011;17(9):693-7.

15. Lökk J. Caregiver strain in Parkinson's disease and the impact of disease duration. Eur J Phys Rehabil Med. 2008;44(1):39-45.

16. McCabe MP, Firth L, O'Connor E. A comparison of mood and quality of life among people with progressive neurological illness and their caregivers. J Clin Psychol Med Settings. 2009;16(4):355-62.

17. Martínez-Martín P, Arroyo S, Rojo-Abuin JM, Rodriguez-Blazquez C, Frades B, Cuesta JP, et al. Burden, perceived health status, and mood among caregivers of Parkinson's disease patients. Mov Disord. 2008;23(12):1673-80.

18. Carter JH, Lyons KS, Stewart BJ, Archbold PG, Scobee R. Does age make a difference in caregiver strain? Comparison of young versus older caregivers in early-stage Parkinson's disease. Mov Disord. 2010; 25(6):724-30.

19. Martínez-Martín P, Benito-Léon J, Alonso F, Catalán MJ, Pondal M, Zamarbide I, et al. Quality of life of caregivers in Parkinson's disease. Qual Life Res. 2005; 14(2):463-72.

20. Schestatsky P, Zanatto VC, Margis R, Chachamovich E, Reche M, Batista RG, et al. Qualidade de vida de uma amostra de pacientes brasileiros portadores da doença de Parkinson e seus cuidadores. Rev Bras Psiquiatr. 2006;28(3):209-11.

21. Brucki S, Nitrini R, Caramelli P, Bertolucci PHF, Okamoto IH. Sugestões para o uso do mini-exame do estado mental no Brasil. Arq Neuropsiquiatr. 2003;61(3-B):777-81.
22. Peto V, Jenkinson C, Fitzpatrick R. Portuguese for Brazil PDQ-39: Parkinson's disease quality of life questionnaire. Version 1.1. Health Services Research Unit: University of Oxford; 1997.

23. Ciconelli RM, Ferraz MB, Santos W, Meinão I, Quaresma MR. Tradução para a língua portuguesa e validação do questionário genérico de avaliação de qualidade de vida SF-36 (Brasil SF-36). Rev Bras Reumatol. 1999;39(3):143-50.

24. Martínez-Martín P, Gil-Nagel A, Morlán Gracia L, Balseiro Gómez J, Martínez-Sarriés J, Bermejo F. Unified Parkinson's disease rating scale characteristics and structure. Mov Disord. 1994;9(1):76-83.

25. Bernal-Pacheco O, Limotai N, Go CL, Fernandez HH. Nonmotor manifestations in Parkinson disease. Neurologist. 2012;18(1):1-16.

26. Kuopio AM, Marttila RJ, Helenius H, Toivonen M, Rinne UK. The quality of life in Parkinson's disease. Mov Disord. 2000;15(2):216-23.

27. Glozman JM. Quality of life of caregivers. Neuropsychol Rev. 2004;14(4):183-96.

28. Filippin NT, Lobo da Costa PH, Mattioli R. Effects of treadmill-walking training with additional body load on quality of life in subjects with Parkinson's disease. Rev Bras Fisioter. 2010;14(4):344-50.

29. Schrag A, Jahanshahi M, Quinn N. What contributes to quality of life in patients with Parkinson's disease? J Neurol Neurosurg Psych. 2000;69(3):308-12.

30. Habermann B, Davis LL. Caring for family with Alzheime's disease and Parkinson's disease: needs, challenges and satisfaction. J Gerontol Nurs. 2005;31(6):49-54.

31. Aarsland D, Larsen JP, Karlsen K, Lim NG, Tandberg E. Mental symptoms in Parkinson's disease are important contributors to caregiver distress. Int J Geriatr Psychiatry. 1999;14(10):866-74.

Recebido: 20/02/2013 Received: 02/20/2013

Aprovado: 09/10/2013 Approved: 10/09/2013 